\title{
Morphologic and molecular analysis of Richter syndrome in chronic lymphocytic leukaemia patients treated with ibrutinib or venetoclax
}

\author{
Ambrus Gángó ${ }^{1,2}$, Richárd Kiss ${ }^{1,2}$, Péter Farkas ${ }^{3}$, Eid Hanna ${ }^{4}$, \\ Judit Demeter $^{4}$, Beáta Deák ${ }^{5}$, Dóra Lévai ${ }^{5}$, Lili Kotmayer ${ }^{1,2}$, \\ Donát Alpár ${ }^{1,2}$, András Matolcsy ${ }^{1,2,6}$, Csaba Bödör ${ }^{1,2}$, Zoltán Mátrai $^{7}$, \\ BOTOND TIMÁR ${ }^{1,2}$ \\ ${ }^{1}$ HCEMM-SE Molecular Oncohematology Research Group, Budapest, Hungary; ${ }^{2} 1$ st \\ Department of Pathology and Experimental Cancer Research, Semmelweis University, \\ Budapest, Hungary; ${ }^{3}$ Department of Internal Medicine and Haematology, Semmelweis Uni- \\ versity, Budapest, Hungary; ${ }^{4}$ Department of Internal Medicine and Oncology, Semmelweis \\ University, Budapest, Hungary; ${ }^{5}$ National Institute of Oncology, Budapest, Hungary; \\ ${ }^{6}$ Department of Laboratory Medicine, Division of Pathology, Karolinska Institutet, Karolinska \\ University Hospital, Stockholm, Sweden, ${ }^{7}$ Central Hospital of Southern Pest, National Institute \\ for Haematology and Infectology, Budapest, Hungary
}

\section{Summary}

Richter syndrome (RS) represents the development of high-grade lymphoma in patients with chronic lymphocytic leukaemia (CLL) or small lymphocytic lymphoma (SLL) and presents a diagnostic and therapeutic challenge with an adverse prognosis. The genetic background and morphology of RS in CLL patients treated with chemoimmunotherapy is extensively characterised; however, our knowledge about RS in patients treated with targeted oral therapies should be extended.

To understand the morphologic and molecular changes leading to RS in CLL patients treated with the Bruton's tyrosine kinase inhibitor, ibrutinib, and the BCL2 inhibitor, venetoclax, sequential samples from six CLL/SLL patients undergoing RS were collected in both the CLL and RS phases.

A detailed immunophenotypic analysis of formalin-fixed, paraffin-embedded tissue specimens of RS phase was performed, followed by extensive molecular characterisation of CLL and RS samples, including the immunoglobulin heavy chain gene $(I G H)$ rearrangement, TP53 mutations, drug-induced resistance mutations in $B T K$ and $B C L 2$ genes and various copy number changes and point mutations detectable with multiplex ligation-dependent probe amplification (MLPA).

Rare, non-diffuse large B-cell lymphoma phenotypes of RS were observed in $3 / 6$ cases, including plasmablastic lymphoma and a transitory entity between diffuse large Bcell lymphoma and classical Hodgkin lymphoma. The majority of cases were clonally related and harboured an unmutated variable region of the immunoglobulin heavy chain gene. Abnormalities affecting the TP53 gene occurred in all patients, and every patient carried at least one genetic abnormality conferring susceptibility to RS. In the background of RS, 2/5 patients treated with ibrutinib showed a BTK C481S resistance mutation. One patient developed a BCL2 G101V mutation leading to venetoclax resistance and $\mathrm{RS}$.

In conclusion, our findings contribute to better understanding of RS pathogenesis in the era of targeted oral therapies. Rare phenotypic variants of RS do occur under the treatment of ibrutinib or venetoclax, and genetic factors leading to RS are similar to those identified in patients treated with chemoimmunotherapy. To our best knowledge, we have reported the first BCL2 G101V mutation in an $\mathrm{RS}$ patient treated with venetoclax.

Key words: Chronic lymphocytic leukaemia; Richter syndrome; ibrutinib; clonal evolution.

Received 4 December 2020, revised 14 April, accepted 18 April 2021 Available online 28 July 2021

\section{INTRODUCTION}

Richter syndrome (RS) represents the development of highgrade lymphoma in the setting of an antecedent or concomitant chronic lymphocytic leukaemia (CLL) or small lymphocytic lymphoma (SLL). ${ }^{1}$ Histologically, two main types of RS are distinguished, diffuse large B-cell lymphoma (DLBCL) and classical Hodgkin lymphoma (cHL), with the former entity comprising the majority (80-90\%) of cases. ${ }^{1}$ Rarely, transformation to plasmablastic lymphoma (PBL), histiocytic sarcoma, lymphoblastic lymphoma and interdigitating dendritic cell sarcoma may occur. ${ }^{2}$ The DLBCL type of RS appears as confluent sheets of large, CD20+ neoplastic B cells with a variable positivity of markers characteristic for the preceding CLL. ${ }^{3}$ The vast majority (90-95\%) of DLBCL type RS cases exhibit a non-germinal centre phenotype (CD10-, MUM1/IRF4+). ${ }^{3-5}$ The cHL variant of RS is characterised by CD30+/CD15+/CD20 - phenotype, with Hodgkin and Reed-Sternberg cells located in the cHLspecific inflammatory background of $\mathrm{T}$ cells, histiocytes, 
eosinophil granulocytes and plasma cells. However, in some CLL cases Hodgkin-like cells with CD30+ expression are in the background of small CLL lymphocytes instead of the reactive cellular milieu of $\mathrm{cHL}$, which is not considered as a 'true' transformation. 6,7

Clonal relatedness is the most important prognostic factor in RS and represents a true transformation in contrast to clonally unrelated lymphomas developing in patients with CLL. While $\sim 80 \%$ of DLBCL-RS is clonally related to the antecedent CLL based on IGHV-D-J rearrangement, only $40-50 \%$ of the cHL-RS arises in a clonally related manner. RS may develop in patients carrying either mutated or unmutated $I G H V$; however, in most of the cases only the latter represents a clonally related transformation. ${ }^{9}$ Although the term 'clonally unrelated RS' is being used widely, these cases are biologically de novo lymphomas with higher response rates to therapy and more favourable prognosis compared to their clonally related counterparts. ${ }^{3-5}$ Patients with clonally unrelated DLBCL-RS have a median survival of $\sim 5$ years, in contrast to the much poorer outcome in clonally related DLBCL-RS (8-16 months). ${ }^{5}$ The outcome of HL-RS patients is more favourable compared to DLBCLRS, but worse than that of the primary classical HL. ${ }^{10} \mathrm{RS}$ developing in patients treated with novel targeted therapies confers a highly adverse prognosis, with only $13 \%$ of patients achieving remission. ${ }^{11,12}$

Over the past years several molecular alterations connected with the development of DLBCL-RS have been identified. Risk factors associated with the high-grade transformation of CLL comprise hereditary single nucleotide polymorphisms (SNP), somatically acquired genetic lesions and clinical features. Hereditary SNPs including BCL2 rs4987852, CD38 rs6449182 and LRP4 rs2306029 predisposing to RS confer a moderate risk via incompletely understood functional mechanisms. ${ }^{13-15}$ Cases of CLL carrying an unmutated $I G H V$ gene are prone to transform into RS, especially when harbouring the IGHV4-39 gene solely or as a part of the stereotyped B-cell receptor subset \#8. ${ }^{4}$ CLL patients with NOTCH1 mutation exhibit a 10-fold higher probability of developing RS compared to their NOTCH1 wild-type counterparts. ${ }^{16-18}$ TP53 defects (mutations or $17 \mathrm{p}$ deletion) are present in $60 \%$ of RS patients, acquired mainly at transformation. ${ }^{5}$ Various genetic events affecting $M Y C$ and $C D K N 2 A$ genes lead to their deregulation and are acquired at the time of transformation. ${ }^{5,19-21}$ Among clinical features, advanced stage (Rai III-IV) and a lymph node size $>3 \mathrm{~cm}$ are the only factors associated with transformation. ${ }^{22-24}$ Predictive markers of CLL progression [decrease in lymphocyte doubling time, bone marrow involvement, elevated $\beta 2$ microglobulin and elevated lactate dehydrogenase (LDH) level] are not associated with RS development, suggesting a biologically distinct way of CLL progression and transformation. ${ }^{24,25}$ Several studies have investigated the impact of the therapy on development of RS. Exposure to fludarabine, a widely used purine analogue was found to be associated with increased risk of RS in some series, ${ }^{26}$ although not in others. ${ }^{25,27}$

Small molecule inhibitors targeting the B-cell receptor (BCR) pathway (especially ibrutinib) and $\mathrm{Bcl} 2$ related apoptosis pathway (venetoclax) have recently revolutionised the therapy of CLL, conferring remarkable outcomes in high-risk as well as in relapsed/refractory patients. ${ }^{28-31}$ In spite of the remarkable results, a proportion of patients undergo progression or Richter transformation (RT) due to the emergence of resistance mutations or additional genetic abnormalities. ${ }^{32,33}$ However, our knowledge about the genetic changes leading to RT in CLL patients treated with novel targeted therapies is incomplete and should be further elucidated.

In this study, we aimed to scrutinise the histological and molecular background of RS in six ibrutinib and/or venetoclax treated patients who underwent high-grade transformation, to gain a better insight into the process leading to the diagnostically and therapeutically challenging phenomenon of RS.

\section{PATIENTS AND METHODS}

The study was conducted in accordance with the Declaration of Helsinki and approved by the Ethics Committee of the Medical Research Council in Hungary.

Sequential samples from six CLL/SLL patients undergoing RT were collected in the CLL phase and at RS diagnosis except for Patient 4 (P4) from whom only a single RS sample was available (although basic cytogenetic characterisation had been performed). The patient cohort consisted of one female and five male patients, their median age at CLL diagnosis was 58 years (38-75 years), and the median follow-up time was 109.5 months (53-355 months). Four patients were treated with ibrutinib, and one patient with venetoclax and ibrutinib+venetoclax combination, respectively. Median time from the initiation of targeted therapy to RT was 20.0 months (2-52 months), the median survival following RT was 1.5 months (0-29 months). Additional clinical data are presented in Table 1 and Fig. 1.

Morphologic and immunophenotypic analysis of RS samples was carried out using formalin-fixed, paraffin-embedded (FFPE) tissue specimens. CD19+ lymphocytes were purified from peripheral blood and bone marrow aspirate with EasySep Human CD19 Positive Selection Kit II (StemCell Technologies, Canada) and DNA was isolated using AllPrep DNA/RNA/ miRNA Universal Kit (Qiagen, Germany). DNA isolation from FFPE tissues was performed with QIAamp DNA FFPE Tissue Kit (Qiagen). After fluorimetric quantification we performed IGHV-IGHD-IGHJ sequencing, nextgeneration sequencing (NGS) of the TP53 gene, multiplex ligationdependent probe amplification (MLPA) targeting relevant abnormalities in CLL and droplet digital polymerase chain reaction (ddPCR) for the detection of resistance mutations against targeted therapies.

The analysis of IGHV-IGHD-IGHJ rearrangements was performed as described by Agathangelidis et al. ${ }^{34}$ DNA amplification with leader primers was followed by bidirectional Sanger sequencing and results were obtained by immunoinformatic analysis using IMGT V-QUEST and ARResT/ AssignSubsets software in concordance with the updated ERIC recommendations. ${ }^{35}$ The whole coding region of TP53 gene including the splice sites was analysed using the SureMASTR TP53 library preparation kit (Agilent, USA) and MiSeq sequencing with a minimum mean coverage of $10,000 \times$ The pathogenicity of TP53 variants was determined using the Seshat tool (https://p53.fr/tp53-database/seshat). Multiplex ligation-dependent probe amplification (MLPA) with SALSA P037 and P038 CLL-specific probemixes (MRC-Holland, The Netherlands) was performed to detect various copy number changes and mutations of great importance in CLL (including deletions of chromosomes 11q, 13q, 17p, trisomy of chromosome 12, and NOTCH1 c.7541_7542delCT, SF3B1 K700E and MYD88 L265P mutations), following the manufacturer's instructions. Resistance mutations in $B T K$ and $B C L 2$ genes were analysed using ddPCR method with mutation specific assays for the detection of the most frequent variants (BTK C481S, dHsaMDS802598840; BCL2 G101V wild-type, dHsaADS52164188; BCL2 G101V mutant type, dHsaADS26433961; BCL2 D103Y wild-type, dHsaADS13624642; BCL2 D103Y mutant type, dHsaADS77894415). Droplet generation and reading was performed using QX200 AutoDG Droplet Digital PCR System (Bio-Rad, USA). The mean sensitivity of the method was $0.01 \%$. 
Table 1 Patient characteristics

\begin{tabular}{|c|c|c|c|c|c|c|c|}
\hline Patient ID & Gender & $\begin{array}{l}\text { Age at CLL } \\
\text { diagnosis, } \\
\text { years }\end{array}$ & $\begin{array}{c}\text { Age } \\
\text { at RS, } \\
\text { years }\end{array}$ & $\begin{array}{l}\text { Time from } \\
\text { CLL diagnosis } \\
\text { to RS, months }\end{array}$ & $\begin{array}{l}\text { Total follow-up } \\
\text { time, months }\end{array}$ & Therapy lines prior to RS & RS morphology \\
\hline P1 & M & 38 & 67 & 355 & 355 & $\begin{array}{l}\text { Chl, Flu, R-B, R-B, R-CVP, IBR, } \\
\text { VEN-R }\end{array}$ & $\begin{array}{l}\text { Null phenotype high-grade } \\
\text { lymphoma }\end{array}$ \\
\hline P2 & $\mathrm{F}$ & 48 & 62 & 178 & 180 & $\begin{array}{l}\text { FC, ofa, R-FC, R-CHOP, R-B, IBR, } \\
\text { R-IBR-VEN }\end{array}$ & DLBCL \\
\hline P4 & M & 67 & 74 & 92 & 93 & R-FC, R-B, IBR & DLBCL \\
\hline P5 & M & 75 & 78 & 41 & 70 & IBR & DLBCL/cHL \\
\hline P6 & M & 75 & 79 & 52 & 53 & R-CVP, CHOP, R, alem, R-B, IBR & Plasmablastic lymphoma \\
\hline
\end{tabular}

alem, alemtuzumab; B, bendamustine; Chl, chlorambucil; cHL, classical Hodgkin lymphoma; CHOP, cyclophosphamide, doxorubicin, vincristine and prednisolone; CLL, chronic lymphocytic leukaemia; COPP/ABV, cyclophosphamide, vincristine, prednisone and procarbazine/doxorubicin, bleomycin and vinblastine; DLBCL, diffuse large B-cell lymphoma; duve, duvelisib; F, female; FC, fludarabine, cyclophosphamide; FCM, fludarabine, cyclophosphamide, mitoxantrone; Flu, fludarabine; IBR, ibrutinib; M, male; ofa, ofatumumab; R, rituximab; R-B, rituximab, bendamustine; R-Chl, rituximab, chlorambucile; R-CHOP, rituximab, cyclophosphamide, doxorubicin, vincristine and prednisolone; R-CVP, rituximab, cyclophosphamide, vincristine, prednisolone; R-FC, rituximab, fludarabine, cyclophosphamide; RS, Richter syndrome; VEN, venetoclax; w, weeks.

\section{RESULTS}

\section{Morphologic patterns of transformation}

All diagnoses for this study were rendered by two independent expert haematopathologists. Three of the six Richter syndrome cases (P2, P3 and P4) were diagnosed as DLBCL showing diffuse proliferation of neoplastic large B lymphocytes with a markedly increased Ki-67 proliferation activity (Table 1).

RS of P1 presented 5 months after venetoclax initiation as a paravertebral mass causing low back pain. The histological examination of the core biopsy specimen revealed a tumour mass with a diffuse pattern composed of polymorphic large cells. The sample was tested with a wide range of immunohistochemical markers. The tumour cells were positive for LCA/CD45 (partial), MUM1, p53, S100 and CD4 stainings with partial Oct-2 and Fascin-1 positivity and a proliferation activity of $60 \%$ by Ki-67. The following markers proved to be negative: CD20, PAX5, CD79a, CD21, CD23, Bcl-6, CD10, CD138, CD30, CD3, CD5, CD7, PD-L1, CD68, ALK1, kappa, lambda, CD15 and melanoma markers (MelanA, HMB45 and SOX10). Based on the partial LCA

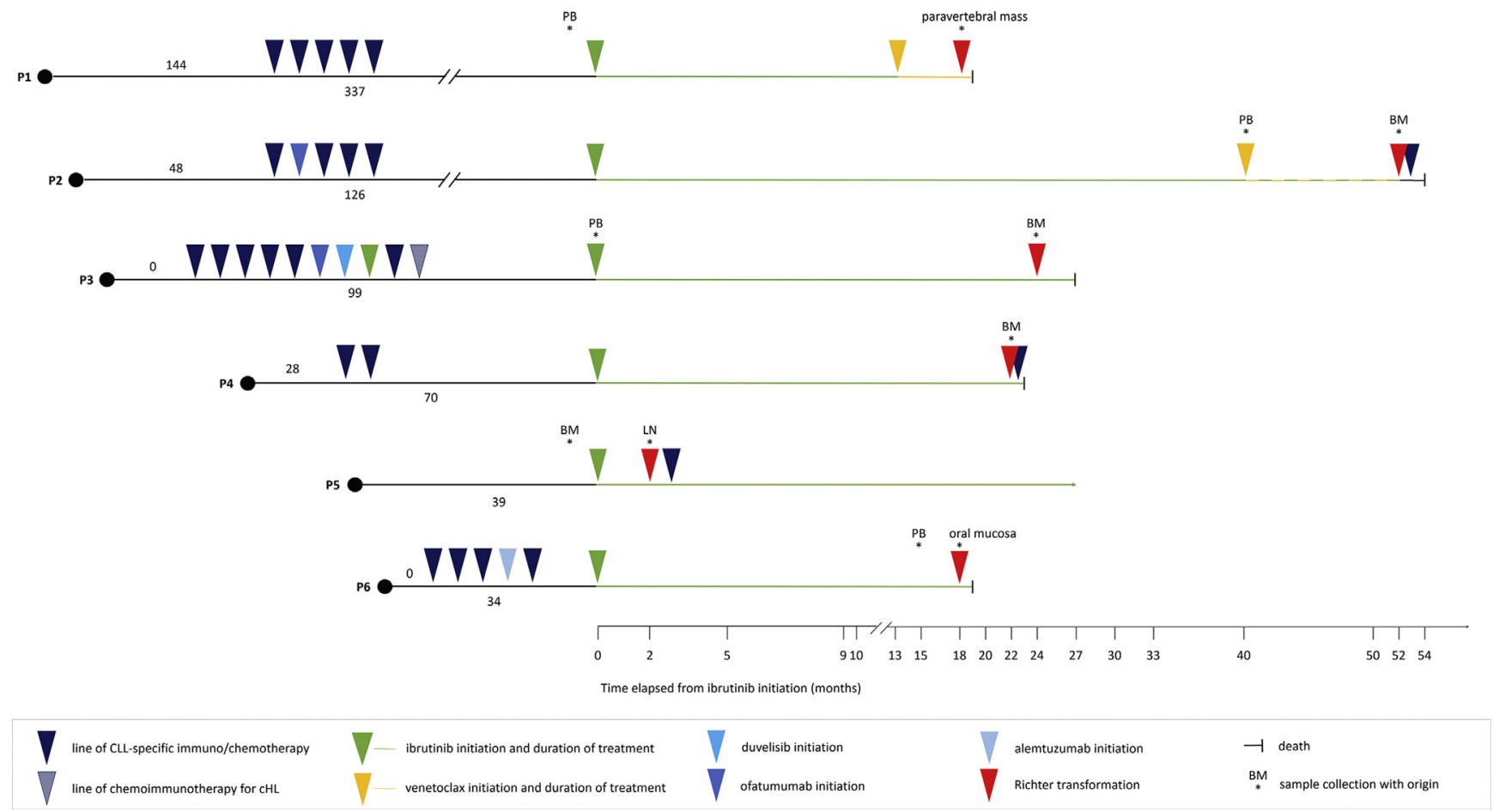

Fig. 1 Timeline and main clinical features of chronic lymphocytic leukaemia (CLL) patients developing Richter syndrome during ibrutinib or venetoclax treatment. The left part of the graph illustrates the clinical course of patients from diagnosis to ibrutinib initiation with lines of therapies. Numbers above the line indicate time to first treatment (TTFT) while numbers below represent time to ibrutinib initiation in months. Patient 2 (P2) received ibrutinib and venetoclax simultaneously for 12 months before transformation. Before long-term ibrutinib therapy, P3 received ibrutinib for 3 weeks, but discontinued due to drug intolerance. P3 developed classical HL simultaneously with CLL and therefore received cHL-directed chemotherapy as well. BM, bone marrow; LN, lymph node; PB, peripheral blood. 

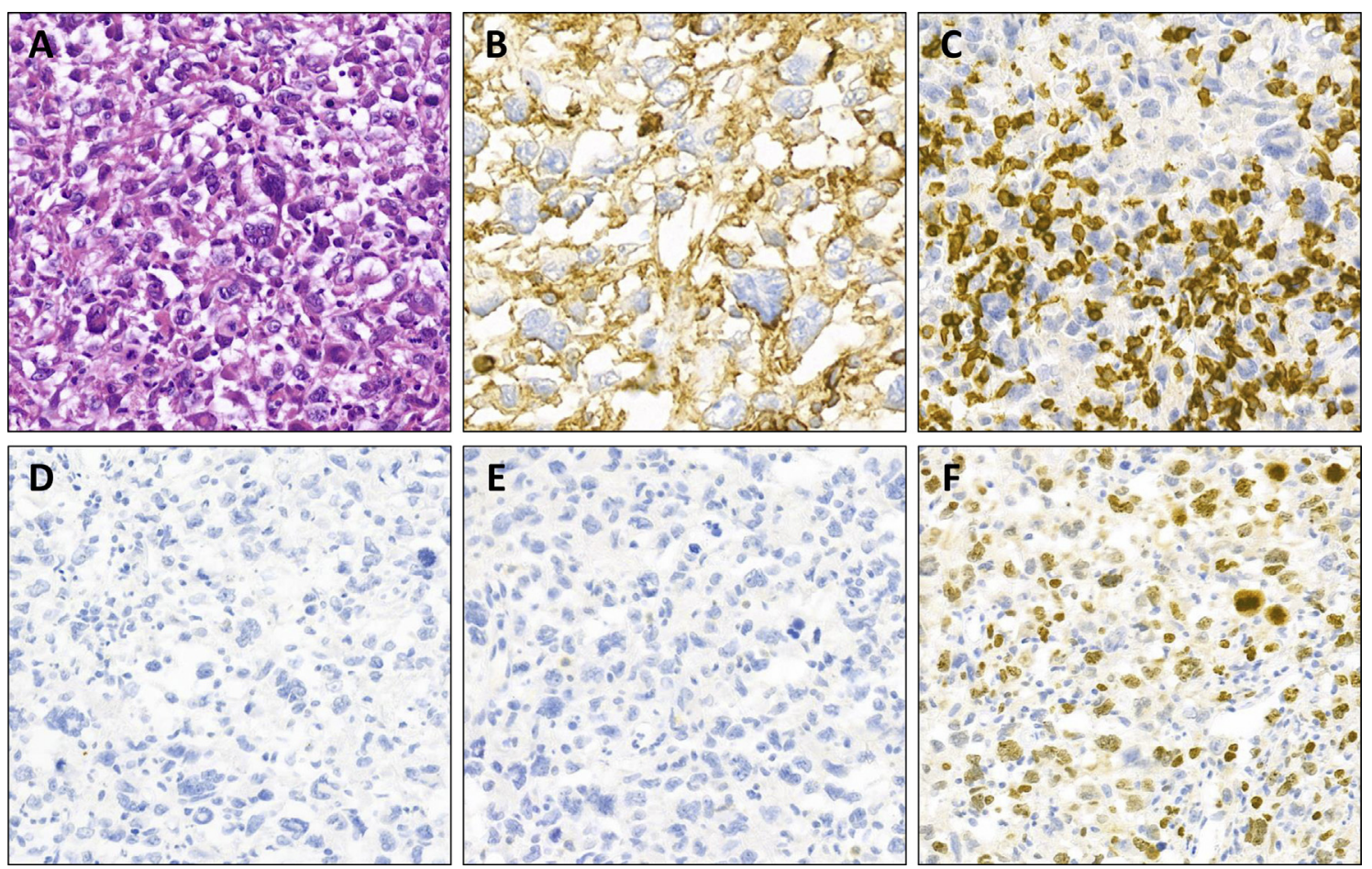

Fig. 2 Richter transformation of chronic lymphocytic leukaemia (CLL) into null-phenotype high-grade lymphoma presented as a paravertebral mass, Patient 1. (A) The neoplastic large cells have lobulated nuclei and prominent nucleoli (H\&E), (B) are of haematopoietic origin (LCA), and show negativity of (D) CD20 and (E) CD30. (C) CD5-positive CLL cells are scattered within the population of large cells. (F) The proliferation activity is approximately 60\% (Ki-67).

and Oct-2 expression, the strong Ki-67 positivity, and the lack of more lineage specific markers (B-cell, T-cell markers, lack of CD30), the initial diagnosis of 'null phenotype' highgrade lymphoma with a morphology resembling Hodgkin lymphoma or anaplastic large cell lymphoma was established, which was later confirmed to be of B-cell origin with monoclonal IgH gene rearrangement (Fig. 2).

P5 experienced a clinically suspicious right inguinal lymph node enlargement 2 months after initiating ibrutinib, concurrently with lymph node regression in other localisations. Histological examination of the core biopsy specimen revealed a diffuse lymphoid infiltrate, and besides small lymphocytes, it consisted mainly of atypical, CD20 and CD30 positive large cells. A proportion of the large cells proved to be multinucleated giant cells. The small cell component showed strong CD5 and weak CD20 positivity. The neoplastic large cells showed a partial expression of C-MYC, a proliferation activity of $60 \%$ by Ki-67 and MUM1 expression of $100 \%$, with negative Bcl-6 staining. Genomic DNA of Epstein-Barr virus (EBV) was not detectable by real-time PCR. In summary, both the morphology and immunophenotype of the large cell component showed mixed features between classical Hodgkin lymphoma and non-germinal centre type DLBCL (Fig. 3).

After 18 months of ibrutinib treatment, P6 showed multiple gingival proliferations which were excised for histological evaluation. Wide subepithelial sheets of tumour cells were observed, and the proliferation activity was approximately $90 \%$ as determined by Ki-67 staining. The tumour cells showed marked LCA, CD79a, CD138 and kappa light chain positivity, while CD20, CD3, CD30, MPO and Pan-CK stainings were negative. Based on this immunophenotype, the diagnosis of plasmablastic lymphoma was established in the patient, without previous immunodeficiency, that is not associated with CLL or CLL-related therapies (e.g., HIV infection) (Fig. 4).

\section{Genetic background of transformation}

\section{Clonal relationship determined by the IGHV-D-J rearrangement}

$I G H V$ analysis was successful in four of six CLL-RS paired samples. Three of the four successfully analysed sample pairs were clonally related (3/6 patients overall), with $I G H V$ mutational status and IGHV-D-J genes being identical. Further details of IGHV-D-J mutation analysis are shown in Table 2.

\section{Chromosome 17p deletions and TP53 mutations}

Abnormalities affecting the key cell cycle regulator TP53 occurred in all patients. TP53 abnormalities were present in all CLL samples and four of six RS samples. Detailed results of TP53 analysis are shown in Fig. 5 and Table 3.

\section{Other genetic aberrations and clinical correlations}

$13 \mathrm{q}$ deletion proved to be the second most frequent chromosomal aberration with three of six affected cases, followed by trisomy 12 ( 2 affected cases). The chromosomal abnormalities occurred in various constellations and were mainly monoallelic (Fig. 5). Using the SALSA P038 CLL-2 probemix, we were able to investigate the presence of three mutations frequently occurring in CLL [SF3B1 c.2098A>G (p.K700E), NOTCH1 c.7541_7542delCT (p.P2514fs) and MYD88 c.794T>C (p.L265P)]. Although cases carrying trisomy 12 are frequently accompanied by NOTCH1 mutations, these two aberrations predisposing to RS occurred 

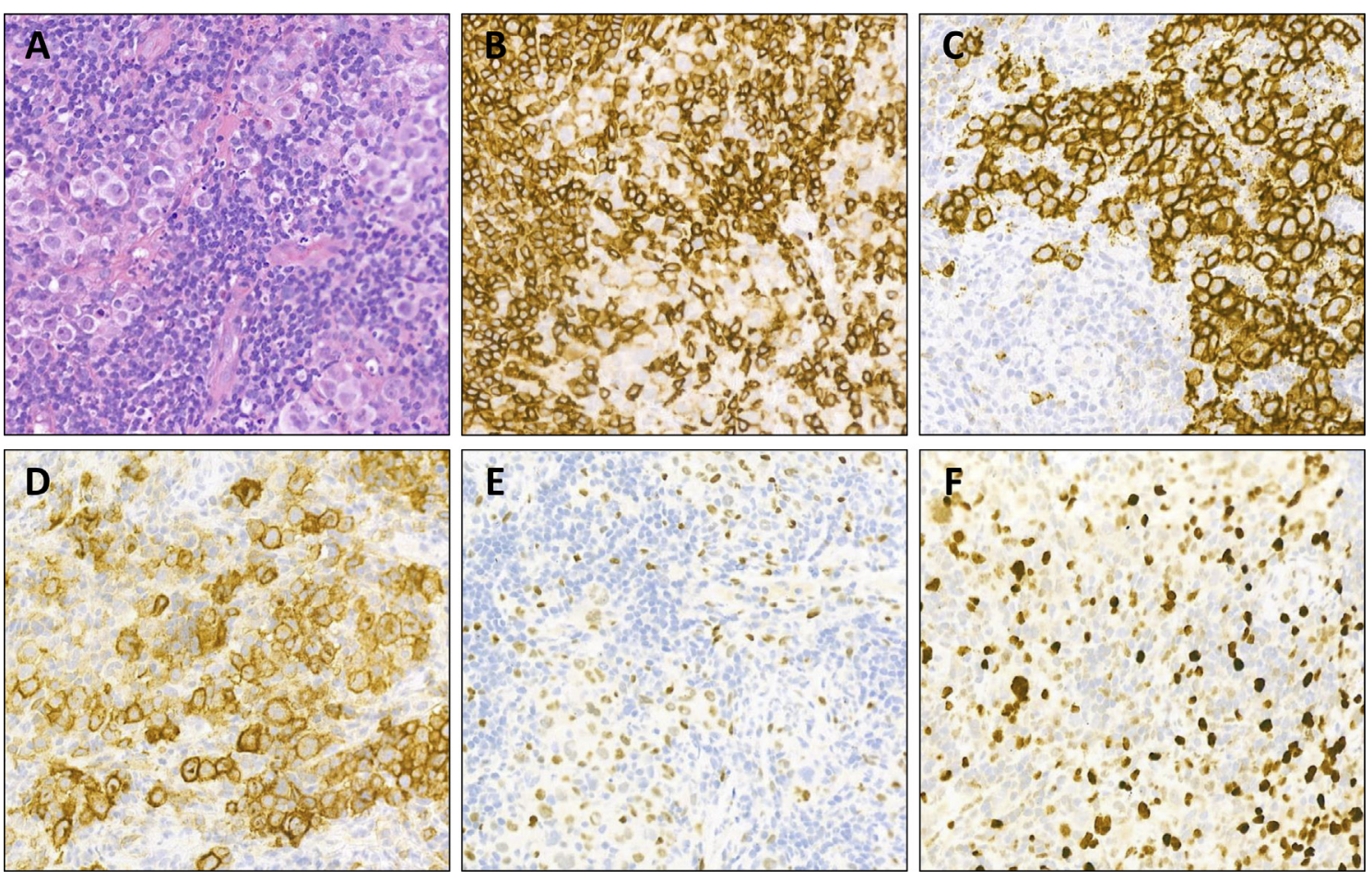

Fig. 3 Richter transformation of chronic lymphocytic leukaemia (CLL) into mixed phenotype B-cell lymphoma with features of diffuse large B-cell lymphoma and classical Hodgkin lymphoma in an inguinal lymph node (Patient 5). (A) The diffuse lymphoid infiltrate effaces the original structure of the lymph node (H\&E). (B) The anaplastic large cell component mixed with the remnant CLL cells (CD5) is positive for (C) CD20, (D) CD30, and (E) C-MYC. (F) The proliferation activity is approximately $60 \%(\mathrm{Ki}-67)$.
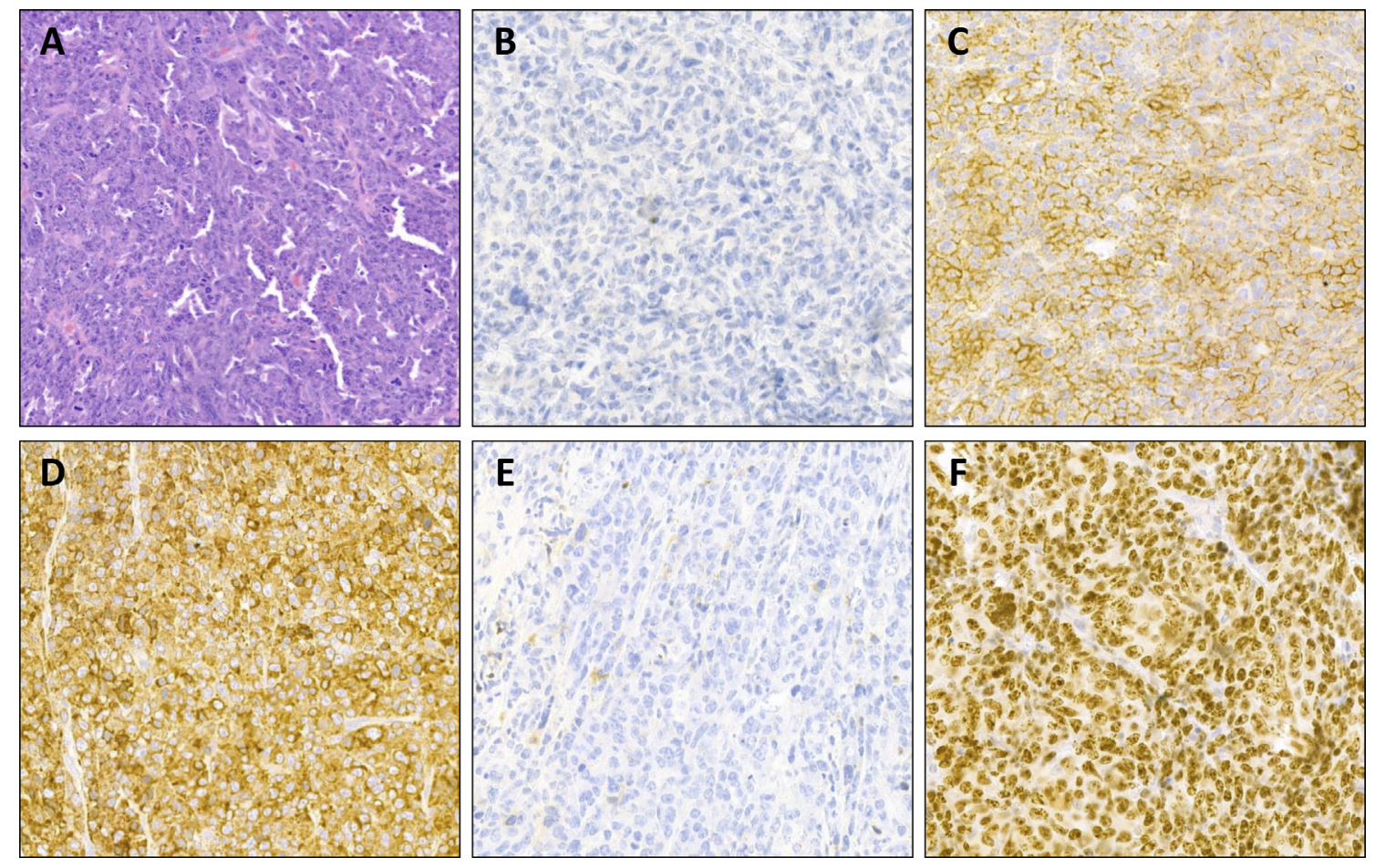

Fig. 4 Richter transformation of CLL into plasmablastic lymphoma (PBL) manifested in the oral mucosa (Patient 6). (A) The neoplastic cells show an immunoblastic morphology with several mitoses (H\&E). (B) The plasmablastic/plasmacytic differentiation is characterised by CD20 negativity and (C) CD138 positivity among other markers. (D) Monoclonal tumour cells show kappa light chain positivity and (E) lambda negativity. (F) The proliferation activity of PBL cells is 90\% (Ki-67).

independently in our cohort. Interestingly, $\mathrm{P} 2$ proved to be the sole patient to harbour a $9 \mathrm{p} 21.3$ deletion, leading to $C D K N 2 A$ and $C D K N 2 B$ loss in both CLL and RS phases. Further details are shown in Fig. 5.
There were remarkable differences among the clinical features of the patients in our cohort, many of which may be linked to certain genetic alterations. P2 carried the largest number of aberrations (6 lesions in CLL and 10 lesions in RS 
Table 2 Clonality analysis

\begin{tabular}{|c|c|c|c|c|c|c|c|c|c|c|c|c|c|}
\hline \multirow[t]{2}{*}{ Patient ID } & \multicolumn{6}{|c|}{ Immunoglobulin heavy chain (CLL) } & \multicolumn{6}{|c|}{ Immunoglobulin heavy chain (RS) } & \multirow[t]{2}{*}{ RS clonality } \\
\hline & $\mathrm{M} / \mathrm{U}$ & $\mathrm{v}$ & $\mathrm{D}$ & $\mathrm{J}$ & CDR3 & Subset & $\mathrm{M} / \mathrm{U}$ & $\mathrm{V}$ & $\mathrm{D}$ & $\mathrm{J}$ & CDR3 & Subset & \\
\hline P1 & M & IGHV4-34*01 F & IGHD6-13*01 F & IGHJ6*02 F & 14 & Unassigned & $\mathrm{M}$ & IGHV1-18*01 F & IGHD2-2*01 F & IGHJ6*02 F & 20 & Unassigned & Unrelated \\
\hline P2 & $\mathrm{U}$ & IGHV3-74*01 F & IGHD5-12*01 F & IGHJ6*02 F & 16 & Unassigned & $\mathrm{U}$ & IGHV3-74*01 F & IGHD5-12*01 F & IGHJ6*02 F & 16 & Unassigned & Related \\
\hline P3 & $\mathrm{U}$ & $\begin{array}{l}\text { IGHV3-33*01 F, } \\
\text { IGHV3-33*06 F }\end{array}$ & IGHD6-13*01 F & IGHJ6*02 F & 23 & Unassigned & $\mathrm{U}$ & $\begin{array}{l}\text { IGHV3-33*01 F, } \\
\text { IGHV3-33*06 F }\end{array}$ & IGHD6-13*01 F & IGHJ6*02 F & 23 & Unassigned & Related \\
\hline P4 & \multicolumn{6}{|c|}{ No data } & $\mathrm{U}$ & $\begin{array}{l}\text { IGHV3-23*01 F, } \\
\text { IGHV3-23D*01 }\end{array}$ & IGHD1-26*01 F & IGHJ4*02 F & 22 & Unassigned & NA \\
\hline P5 & $\mathrm{U}$ & $\begin{array}{l}\text { IGHV1-69*01 F, } \\
\text { IGHV1-69*01D F }\end{array}$ & IGHD3-16*02 F & IGHJ $3 * 02 \mathrm{~F}$ & 21 & CLL\#6 & $\mathrm{U}$ & $\begin{array}{l}\text { IGHV1-69*01 F, } \\
\text { IGHV1-69*01D F }\end{array}$ & IGHD3-16*02 F & IGHJ3*02 F & 21 & CLL\#6 & Related \\
\hline P6 & $\mathrm{U}$ & IGHV1-3*01 F & IGHD6-19*01 F & IGHJ $4 * 02 \mathrm{~F}$ & 13 & CLL\#1 & No d & & & & & & NA \\
\hline
\end{tabular}

CDR3, complementarity determining region 3; CLL, chronic lymphocytic leukaemia; D, diversity; J, joining; M, mutated; NA, not applicable; RS, Richter syndrome; U, unmutated; V, variable.

Table 3 TP53 mutations identified in the paired samples

\begin{tabular}{|c|c|c|c|c|c|c|c|c|c|c|}
\hline \multirow[t]{2}{*}{ Patient ID } & \multicolumn{5}{|c|}{ TP53 mutation (CLL) } & \multicolumn{5}{|c|}{ TP53 mutation (RS) } \\
\hline & cDNA change & Amino acid change & VAF (\%) & COSMIC ID & Clinical significance & cDNA change & Amino acid change & $\operatorname{VAF}(\%)$ & COSMIC ID & Clinical significance \\
\hline \multirow[t]{5}{*}{ P1 } & c.533delA & p.H178Pfs*69 & $1.8 \%$ & COSM6960219 & Deleterious & Negative & & & & \\
\hline & c.533delinsCC & p.H178Pfs*3 & $1.3 \%$ & Novel & Probably deleterious & & & & & \\
\hline & c. $536 \mathrm{~A}>\mathrm{G}$ & p.H179R & $3.2 \%$ & COSM10889 & Pathogenic & & & & & \\
\hline & c. $713 \mathrm{G}>\mathrm{A}$ & p.C238Y & $1.6 \%$ & COSM11059 & Pathogenic & & & & & \\
\hline & c. $814 \mathrm{G}>\mathrm{A}$ & p.V272M & $22.0 \%$ & COSM10891 & Pathogenic & & & & & \\
\hline \multirow[t]{2}{*}{ P2 } & c. $425 \mathrm{C}>\mathrm{T}$ & p.P142L & * & COSM43583 & VUS & c. $595 \mathrm{G}>\mathrm{T}$ & p.G199* & $2.9 \%$ & COSM44537 & Deleterious \\
\hline & & & & & & c. $611 \mathrm{~A}>\mathrm{C}$ & p.E204A & $1.1 \%$ & COSM46223 & VUS \\
\hline \multirow[t]{4}{*}{ P3 } & c. $472 \mathrm{C}>\mathrm{G}$ & p.R158G & $4.6 \%$ & COSM11087 & Deleterious & c. $517 \mathrm{G}>\mathrm{T}$ & p.V173L & $1.6 \%$ & COSM43559 & Pathogenic \\
\hline & c. $503 \mathrm{~A}>\mathrm{G}$ & p.H168R & $2.1 \%$ & COSM43545 & Deleterious & & & & & \\
\hline & c.652_654delGTG & p.V218del & $3.7 \%$ & COSM6496 & VUS & & & & & \\
\hline & c. $747 \mathrm{G}>\mathrm{T}$ & p.R249S & $12.0 \%$ & COSM10817 & Pathogenic & & & & & \\
\hline P4 & No data & & & & & c. $466 \mathrm{C}>\mathrm{G}$ & p.R156G & $*$ & COSM45154 & Probably deleterious \\
\hline P5 & Negative & & & & & c. $584 \mathrm{~T}>\mathrm{C}$ & p.I195T & $*$ & COSM11089 & Pathogenic \\
\hline P6 & c.771_777delGGAAGAC & p.E258Pfs*85 & $8.5 \%$ & Novel & Probably deleterious & Negative & & & & \\
\hline
\end{tabular}

VAF, variant allele frequency; VUS, variant of unknown significance; *, samples analysed by Sanger sequencing outside of the setting of this study

VAF must be over the $20 \%$ sensitivity of Sanger sequencing, but its exact value is unknown.

COSMIC IDs are given according to COSMIC version v91. 
phase), resulting in a complex karyotype and genomic instability leading to a highly aggressive large cell transformation of CLL resembling acute leukaemia. P1 harbouring $13 \mathrm{q} \mathrm{del}$ and $I G H V$-M (germline homology of $96.14 \%$ ) was observed for 12 years after diagnosis with no need of treatment, and RS proved to be clonally unrelated due to the use of different $I G H$ genes, but still being $I G H V$-M (germline homology of $93.51 \%$ ). Although trisomy 12 confers an intermediate prognosis, the unmutated IGHV gene with the PBL phenotype and the presence of stereotyped BCR subset \#1 may lead to a particularly dismal outcome as documented in the case of P6. The total follow-up time (53 months) of P6 is the shortest in our cohort; the patient had to be treated immediately after diagnosis and PBL transformation occurred 18 months after ibrutinib initiation (Fig. 1). P1 and P6 succumbed to RS after a total follow-up time of 355 and 53 months, respectively (Fig. 1).

P5 harboured an adverse SF3B1 mutation with a VAF of $3 \%$ and an $I G H V-U$ genotype. Although generally stereotyped BCRs confer a less favourable outcome in the IGHV-U patients, subset \#6 carried by P5 did not have such a negative impact on survival as subset \#1 in P6. At transformation, P5 continued ibrutinib therapy with the addition of 6 cycles of rituximab-bendamustine and is still in remission 29 months after developing clonally related RS (follow-up 70 months).

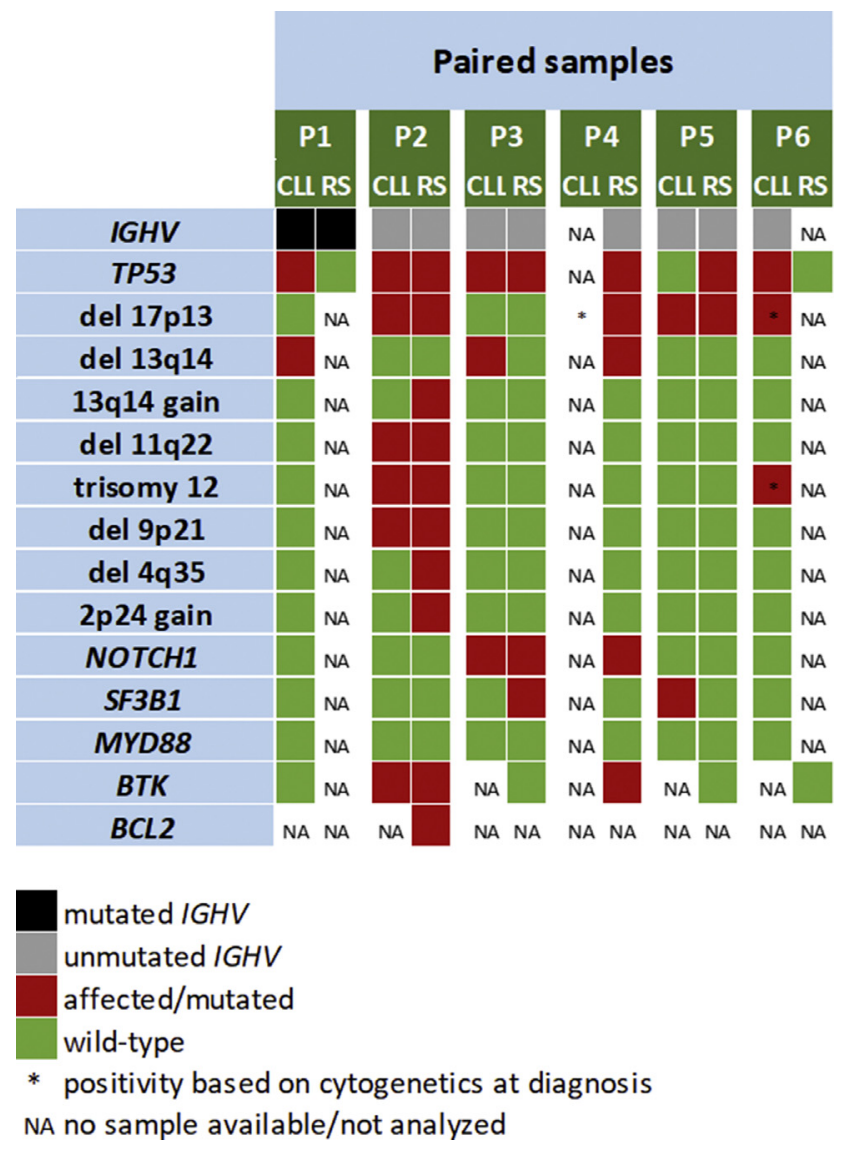

Fig. 5 Heat map displaying the $I G H V$ mutational status, chromosomal aberrations and mutations identified across the six patients. Chronic lymphocytic leukaemia (CLL) sample from Patient 4 (P4) was not available. NA (not applicable) denotes absent data due to sample unavailability, unsuccessful or unreasonable analysis (e.g., BCL2 analysis in venetoclax-naïve patients).

\section{Ibrutinib/venetoclax resistance mutations}

Two of five RS samples analysed showed a BTK C481S ibrutinib resistance mutation ( $\mathrm{P} 4$ and $\mathrm{P} 2)$. One patient carried a $B C L 2 \mathrm{G} 101 \mathrm{~V}$ venetoclax resistance mutation (P2), the RS sample of the other patient undergoing venetoclax therapy (P1) was not available in the required amount for ddPCR analysis. P4 developed a BTK C481S mutation with a variant allele frequency (VAF) of $0.21 \%$ that was detectable in the RS phase 22 months after ibrutinib initiation. P2 was treated with ibrutinib until she progressed 40 months after initiating ibrutinib. After progression, venetoclax and rituximab were added to ibrutinib and this combination was administered for 12 months until RT occurred. CLL sample of P2 was collected at progression and showed a BTK C481S mutation with a VAF of $11.9 \%$. One year later, at the time of transformation, the BTK C481S VAF decreased to $0.95 \%$ while the BCL2 G101V mutation emerged with a VAF of $4.6 \%$.

\section{DISCUSSION}

The BTK inhibitor ibrutinib and BCL2 inhibitor venetoclax have revolutionised the treatment of CLL over recent years, especially in the setting of relapsing/refractory or TP53 mutated disease. However, clonal evolution initiated by conventional chemoimmunotherapy continues under the selective pressure exerted by targeted therapies, and drives progression, relapse and transformation. RS developing under chemoimmunotherapy was studied excessively, but the genetic and morphologic characterisation of ibrutinib- or venetoclax-induced RT needs further elucidation.

Although our knowledge is rapidly growing on the molecular and radiological features of RS, histological examination has an outstanding impact on diagnosis establishment and the morphology correlates frequently with molecular and clinical features. In the era of cytotoxic therapies, CLL transformation to PBL occurred extremely rarely. ${ }^{36}$ However, in a cohort of four post-ibrutinib RS cases reported by Chan et al., $50 \%$ of the patients showed PBL morphology, ${ }^{37}$ and a further similar case was reported by others. ${ }^{38}$ The molecular mechanism underlying PBL transformation of ibrutinib treated CLL is unclear. Most of the PBL cases are extranodal and arise de novo in association with immunodeficiency or EBV positivity. Secondary cases show monoclonal $I G H^{39}$ and $M Y C$ rearrangement, ${ }^{40}$ and cases with trisomy 12 have also been reported ${ }^{41}$ similar to our P6. Considering that BTK is downregulated in plasma cells, ${ }^{42}$ plasmablastic transformation of ibrutinib treated CLL may be an escape mechanism to overcome BTK inhibition ${ }^{37}$ without developing a resistance mutation. In line with this theory, P6 in our cohort underwent RT characterised by PBL morphology without the canonical BTK C481S resistance mutation, similarly to three previously published cases. ${ }^{37,38}$

The development of ibrutinib resistance mutations in BTK and PLCG2 genes is the leading cause of CLL progression. ${ }^{38,43} \mathrm{RS}$ during ibrutinib treatment occurs in 5.0-7.3\% of patients within 17-24 months after ibrutinib initiation, as reviewed by Lampson et al. ${ }^{44}$ The mean period ( 20 months) from ibrutinib start to RT in our cohort is in line with these findings. BTK or PLCG 2 mutations occur in $~ 80 \%$ of patients with CLL progression, but in only $\sim 40 \%$ of RS cases. ${ }^{4,46}$ This difference may be explained by the underestimated mutation frequency because of the limited availability of RS 
tissues compared to peripheral blood, or more likely by biologically distinct ways of CLL progression and RS. In our cohort, two of five patients harboured a BTK C481S mutation among those who underwent RT during ibrutinib treatment. This prevalence corresponds to the literature; however, we should note that non-C481S BTK mutations or PLCG2 mutations were not analysed in this study.

RS during venetoclax therapy occurred in two patients in our cohort 8.5 months after therapy initiation. Interestingly, the high frequency of RS observed in heavily pretreated patients ${ }^{47}$ may not correspond to the frequency of RS in a less heavily pretreated cohort. ${ }^{48}$ Refractoriness to fludarabine and complex karyotype are the main risk factors of early progression in venetoclax treated patients, ${ }^{11}$ the latter presumably being responsible for the early RS and aggressive clinical course of $\mathrm{P} 2$ in our cohort. Acquired abnormalities in the $B T G 1, T P 53, C D K N 2 A / B, S F 3 B 1$ and $B R A F$ genes have been reported in the background of CLL progression and RS in venetoclax-treated patients. ${ }^{49}$ To our best knowledge, $B C L 2$ mutations have not yet been reported in $\mathrm{RS} ;{ }^{50}$ therefore, this study might be the first to reveal a BCL2 G101V resistance mutation in RS developing during venetoclax therapy. However, as remnant CLL clones were observed besides the DLBCL component in the patient's bone marrow biopsy, a CLL origin of $B C L 2$ G101V mutation could not be excluded.

Given that some genetic lesions present in CLL phase were absent in RS phase, they could have been replaced by CLL subclones with a higher potential towards transformation. Indeed, 13q deletion observed in CLL phase of P3 has been eliminated, while an $S F 3 B 1 \mathrm{~K} 700 \mathrm{E}$ mutation predisposing to RS emerged. Furthermore, subclonal dynamics affecting TP53 mutations has also been detected. All six patients carried TP53 defect (17p deletion, TP53 mutation or both). In line with our previous findings in ibrutinib-treated CLL, ${ }^{43}$ the majority of TP53 mutated subclones have been eliminated under the selective pressure of ibrutinib. In the CLL phases of P1 and P6, six TP53 mutations were identified altogether, and all of them were absent in the RS phase. The four TP53 mutations of the CLL sample of P3 were replaced with only one mutation detected in the RS phase.

In summary, our findings support the importance of morphologic and molecular analysis in Richter transformation of CLL in the era of oral targeted therapies. Since all patients except for one had received multiple lines of cytotoxic chemotherapy before initiating targeted therapies, the morphologic and genetic changes observed do not necessarily represent the features of cytotoxic therapy-naïve RS patients. Every patient in our cohort carried at least one genetic aberration conferring susceptibility to RS, with the predisposing factors in ibrutinib/venetoclax-treated patients being the same as identified in patients treated with chemoimmunotherapy (unmutated $I G H V$, stereotyped BCRs, TP53 defect, trisomy $12, C D K N 2 A / B$ loss, $M Y C$ aberrations, NOTCH1 and SF3B1 mutations). BTK or BCL2 mutations play a less prominent role in RS pathogenesis compared to CLL progression or relapse. To our best knowledge, we have reported the first $B C L 2 \mathrm{G} 101 \mathrm{~V}$ mutation in an RS patient treated with venetoclax.

Acknowledgements: This work was funded by the KH17_126718, K21_137948, FK20_134253 and K_16
\#119950 grants of the Hungarian National Research, Development and Innovation Office (NKFIH), the EU's Horizon 2020 research and innovation program under grant agreement no. 739593, and the János Bolyai Research Scholarship (BO/00320/18/5) of the Hungarian Academy of Sciences, the ÚNKP-19-3-I-SE-52 and ÚNKP-20-5-SE-22 grants of the Ministry of Innovation and Technology, and the Higher Education Institutional Excellence Programme of the Ministry of Human Capacities in Hungary, within the framework of the Molecular Biology thematic programme of the Semmelweis University and the ELIXIR Hungary.

Conflicts of interest and sources of funding: The authors state that there are no conflicts of interest to disclose.

Address for correspondence: Dr Botond Timár, HCEMM-SE Molecular Oncohematology Research Group, 1st Department of Pathology and Experimental Cancer Research, Semmelweis University, Üllöi út 26, H1085, Budapest, Hungary. E-mail: timar.botond@med.semmelweis-univ.hu

\section{References}

1. Campo E, Ghia P, Montserrat E, et al. Chronic lymphocytic leukemia/ small lymphocytic lymphoma. In: Swerdlow SH, Campo E, Harris NL, editors. World Health Organization Classification of Tumours of Hematopoietic and Lymphoid Tissues. Lyon: IARC, 2017; 180-2.

2. Jain P, O'Brien S. Richter's transformation in chronic lymphocytic leukemia. Oncology 2012; 26: 1146-52.

3. Mao Z, Quintanilla-Martinez L, Raffeld M, et al. IgVH mutational status and clonality analysis of Richter's transformation: diffuse large Bcell lymphoma and Hodgkin lymphoma in association with B-cell chronic lymphocytic leukemia (B-CLL) represent 2 different pathways of disease evolution. Am J Surg Pathol 2007; 31: 1605-14.

4. Rossi D, Spina V, Cerri M, et al. Stereotyped B-cell receptor is an in dependent risk factor of chronic lymphocytic leukemia transformation to Richter syndrome. Clin Cancer Res 2009; 15: 4415-22.

5. Rossi D, Spina V, Deambrogi C, et al. The genetics of Richter syndrome reveals disease heterogeneity and predicts survival after transformation. Blood 2011; 117: 3391-401.

6. Bockorny B, Codreanu I, Dasanu CA. Hodgkin lymphoma as Richter transformation in chronic lymphocytic leukaemia: a retrospective analysis of world literature. Br J Haematol 2012; 156: 50-66.

7. Tsimberidou AM, O'Brien S, Kantarjian HM, et al. Hodgkin transformation of chronic lymphocytic leukemia: the MD Anderson Cancer Center experience. Cancer 2006; 107: 1294-302.

8. Rossi D, Gaidano G. Richter syndrome: pathogenesis and management. Semin Oncol 2016; 43: 311-9.

9. Timár B, Fülöp Z, Csernus B, et al. Relationship between the mutational status of $\mathrm{VH}$ genes and pathogenesis of diffuse large B-cell lymphoma in Richter's syndrome. Leukemia 2004; 18: 326-30.

10. Rossi D, Spina V, Gaidano G. Biology and treatment of Richter syndrome. Blood 2018; 131: 2761-72.

11. Anderson MA, Tam C, Lew TE, et al. Clinicopathological features and outcomes of progression of CLL on the BCL2 inhibitor venetoclax. Blood 2017; 129: 3362-70.

12. Jain P, Thompson PA, Keating M, et al. Long-term outcomes for patients with chronic lymphocytic leukemia who discontinue ibrutinib. Cancer 2017; 123: 2268-73.

13. Aydin S, Rossi D, Bergui L, et al. CD38 gene polymorphism and chronic lymphocytic leukemia: a role in transformation to Richter syndrome? Blood 2008; 111: 5646-53.

14. Parikh SA, Shanafelt TD. Risk factors for Richter syndrome in chronic lymphocytic leukemia. Curr Hematol Malig Rep 2014; 9: 294-9.

15. Rasi S, Spina V, Bruscaggin A, et al. A variant of the LRP4 gene affects the risk of chronic lymphocytic leukaemia transformation to Richter syndrome. Br J Haematol 2011; 152: 284-94.

16. Rossi D, Rasi S, Fabbri G, et al. Mutations of NOTCH1 are an independent predictor of survival in chronic lymphocytic leukemia. Blood 2012; 119: 521-9.

17. Rossi D, Rasi S, Spina V, et al. Different impact of NOTCH1 and SF3B1 mutations on the risk of chronic lymphocytic leukemia transformation to Richter syndrome. Br J Haematol 2012; 158: 426-9. 
18. Villamor N, Conde L, Martinez-Trillos A, et al. NOTCH1 mutation identify a genetic subgroup of chronic lymphocytic leukemia patients with high risk of transformation and poor outcome. Leukemia 2013; 27: 1100-6.

19. Chigrinova E, Rinaldi A, Kwee I, et al. Two main genetic pathways lead to the transformation of chronic lymphocytic leukemia to Richter syndrome. Blood 2013; 122: 2673-82

20. Fabbri G, Khiabanian H, Holmes AB, et al. Genetic lesions associated with chronic lymphocytic leukemia transformation to Richter syndrome. J Exp Med 2013; 210: 2273-88.

21. Rossi D, Berra E, Cerri M, et al. Aberrant somatic hypermutation in transformation of follicular lymphoma and chronic lymphocytic leukemia to diffuse large B-cell lymphoma. Haematologica 2006; 91: 1405-9.

22. Maurer C, Langerbeins $\mathrm{P}, \mathrm{B}$ ahlo J, et al. Effect of first-line treatment on second primary malignancies and Richter's transformation in patients with CLL. Leukemia 2016; 30: 2019-25.

23. Parikh SA, Kay NE, Shanafelt TD. How we treat Richter syndrome. Blood 2014; 123: 1647-57.

24. Rossi D, Cerri M, Capello D, et al. Biological and clinical risk factors of chronic lymphocytic leukaemia transformation to Richter syndrome. $\mathrm{Br}$ J Haematol 2008; 142: 202-15.

25. Parikh SA, Rabe KG, Call TG, et al. Diffuse large B-cell lymphoma (Richter syndrome) in patients with chronic lymphocytic leukaemia (CLL): a cohort study of newly diagnosed patients. Br J Haematol 2013; 162: $774-82$.

26. Maddocks-Christianson K, Slager SL, Zent CS, et al. Risk factors for development of a second lymphoid malignancy in patients with chronic lymphocytic leukaemia. Br J Haematol 2007; 139: $398-404$.

27. Catovsky D, Richards S, Matutes E, et al. Assessment of fludarabine plus cyclophosphamide for patients with chronic lymphocytic leukaemia (the LRF CLL4 Trial): a randomised controlled trial. Lancet 2007; 370 230-9.

28. Ahn IE, Farooqui MZH, Tian X, et al. Depth and durability of response to ibrutinib in CLL: 5-year follow-up of a phase 2 study. Blood 2018; 131: $2357-66$

29. Burger JA, Tedeschi A, Barr PM, et al. Ibrutinib as initial therapy for patients with chronic lymphocytic leukemia. N Engl J Med 2015; 373: $2425-37$.

30. Byrd JC, Brown JR, O'Brien S, et al. Ibrutinib versus ofatumumab in previously treated chronic lymphoid leukemia. N Engl J Med 2014; 371 $213-23$.

31. Farooqui MZ, Valdez J, Martyr S, et al. Ibrutinib for previously un treated and relapsed or refractory chronic lymphocytic leukaemia with TP53 aberrations: a phase 2, single-arm trial. Lancet Oncol 2015; 16 $169-76$

32. Furman RR, Cheng $\mathrm{S}$, Lu P, et al. Ibrutinib resistance in chronic lym phocytic leukemia. N Engl J Med 2014; 370: 2352-4.

33. Woyach JA, Furman RR, Liu TM, et al. Resistance mechanisms for the Bruton's tyrosine kinase inhibitor ibrutinib. N Engl J Med 2014; 370: 2286-94.

34. Agathangelidis A, Sutton LA, Hadzidimitriou A, et al. Immunoglobulin gene sequence analysis in chronic lymphocytic leukemia: from patient material to sequence interpretation. J Vis Exp 2018; 141: e57787.
35. Rosenquist R, Ghia P, Hadzidimitriou A, et al. Immunoglobulin gene sequence analysis in chronic lymphocytic leukemia: updated ERIC recommendations. Leukemia 2017; 31: 1477-81.

36. Evans AG, Rothberg PG, Burack WR, et al. Evolution to plasmablastic lymphoma evades CD19-directed chimeric antigen receptor T cells. $\mathrm{Br} J$ Haematol 2015; 171: 205-9.

37. Chan KL, Blombery $\mathrm{P}$, Jones $\mathrm{K}$, et al. Plasmablastic Richter transformation as a resistance mechanism for chronic lymphocytic leukaemia treated with BCR signalling inhibitors. Br J Haematol 2017; 177: $324-8$.

38. Maddocks KJ, Ruppert AS, Lozanski G, et al. Etiology of ibrutinib therapy discontinuation and outcomes in patients with chronic lymphocytic leukemia. JAMA Oncol 2015; 1: 80-7.

39. Martinez D, Valera A, Perez NS, et al. Plasmablastic transformation of low-grade B-cell lymphomas: report on 6 cases. Am J Surg Pathol 2013 37: $272-81$

40. Pan Z, Xie Q, Repertinger S, Richendollar BG, Chan WC, Huang Q. Plasmablastic transformation of low-grade CD5+ B-cell lymphoproliferative disorder with MYC gene rearrangements. Hum Pathol 2013; 44: $2139-48$.

41. Gasljevic G, Grat M, Kloboves Prevodnik V, et al. Chronic lymphocytic leukemia with divergent Richter's transformation into a clonally related classical Hodgkin's and plasmablastic lymphoma: a case report. Case Rep Oncol 2020; 13: 120-9.

42. de Weers M, Verschuren MC, Kraakman ME, et al. The Bruton's tyrosine kinase gene is expressed throughout B cell differentiation, from early precursor B cell stages preceding immunoglobulin gene rearrangement up to mature B cell stages. Eur J Immunol 1993; 23: 3109-14.

43. Gango A, Alpar D, Galik B, et al. Dissection of subclonal evolution by temporal mutation profiling in chronic lymphocytic leukemia patients treated with ibrutinib. Int J Cancer 2020; 146: 85-93.

44. Lampson BL, Brown JR. Are BTK and PLCG2 mutations necessary and sufficient for ibrutinib resistance in chronic lymphocytic leukemia? Exp Rev Hematol 2018; 11: 185-94.

45. Kadri S, Lee J, Fitzpatrick C, et al. Clonal evolution underlying leukemia progression and Richter transformation in patients with ibrutinibrelapsed CLL. Blood Adv 2017; 1: 715-27.

46. Woyach JA, Ruppert AS, Guinn D, et al. BTK(C481S)-mediated resistance to ibrutinib in chronic lymphocytic leukemia. J Clin Onco 2017; 35: $1437-43$

47. Roberts AW, Davids MS, Pagel JM, et al. Targeting BCL2 with venetoclax in relapsed chronic lymphocytic leukemia. $N$ Engl J Med 2016; 374: 311-22.

48. Seymour JF, Kipps TJ, Eichhorst B, et al. Venetoclax-rituximab in relapsed or refractory chronic lymphocytic leukemia. $N$ Engl $J$ Med 2018; 378: 1107-20.

49. Herling CD, Abedpour N, Weiss J, et al. Clonal dynamics towards the development of venetoclax resistance in chronic lymphocytic leukemia. Nat Commun 2018; 9: 727.

50. Blombery $\mathrm{P}$, Anderson MA, Gong JN, et al. Acquisition of the recurrent Gly101Val mutation in BCL2 confers resistance to venetoclax in patients with progressive chronic lymphocytic leukemia. Cancer Discov 2019; 9: 342-53. 